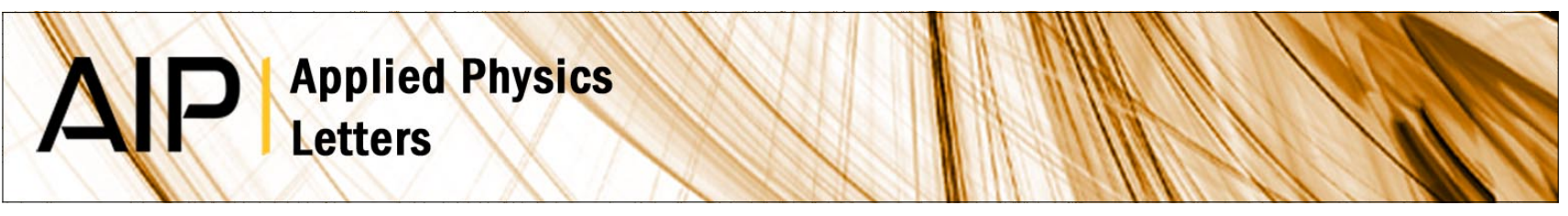

\title{
Anharmonic effects in ZnO optical phonons probed by Raman spectroscopy
}

Marius Millot, Ramon Tena-Zaera, Vicente Munoz-Sanjose, Jean-Marc Broto, and Jesus Gonzalez

Citation: Appl. Phys. Lett. 96, 152103 (2010); doi: 10.1063/1.3387843

View online: http://dx.doi.org/10.1063/1.3387843

View Table of Contents: http://apl.aip.org/resource/1/APPLAB/v96/i15

Published by the American Institute of Physics.

\section{Additional information on Appl. Phys. Lett.}

Journal Homepage: http://apl.aip.org/

Journal Information: http://apl.aip.org/about/about_the_journal

Top downloads: http://apl.aip.org/features/most_downloaded

Information for Authors: http://apl.aip.org/authors

\section{ADVERTISEMENT}

\section{AIP | Applied Physics Letters}

Accepting Submissions in

Biophysics and Bio-Inspired Systems 


\title{
Anharmonic effects in ZnO optical phonons probed by Raman spectroscopy
}

\author{
Marius Millot, ${ }^{1,2, a)}$ Ramon Tena-Zaera, ${ }^{3, b)}$ Vicente Munoz-Sanjose, ${ }^{3}$ Jean-Marc Broto, ${ }^{1}$ \\ and Jesus Gonzalez ${ }^{2}$ \\ ${ }^{1}$ Laboratoire National des Champs Magnétiques Intenses (LNCMI), CNRS UPR 3228, Université de \\ Toulouse, 143 Avenue de Rangueil, 31400 Toulouse, France \\ ${ }^{2}$ DCITIMAC-Malta Consolider Team, Universidad de Cantabria, Avenida Los Castros S/N, 39005 \\ Santander, Cantabria, Spain and Centro de Estudios de Semiconductores, Universidad de los Andes, Mérida \\ 5201, Venezuela \\ ${ }^{3}$ Departamento de Fisica Aplicada y Electromagnetismo, Universitat de Valencia, C/Dr. Moliner 50, 46100 \\ Burjassot, Spain
}

(Received 3 February 2010; accepted 23 March 2010; published online 12 April 2010)

\begin{abstract}
We report Raman spectroscopy measurements on $\mathrm{ZnO}$ crystals grown by the vapor transport method and annealed. Vacuum annealing is found to yield single crystals with ultra low density of defects. We focus on the optical $E_{2}$ phonon linewidth temperature dependence in the $10-500 \mathrm{~K}$ range. The linewidth decrease at low temperature is analyzed and discussed in the light of anharmonic up- and down-conversion processes, unveiling strongly different behaviors for the two $E_{2}$ phonons. (C) 2010 American Institute of Physics. [doi:10.1063/1.3387843]
\end{abstract}

Nowadays considered to be a promising basis for diluted magnetic semiconductor, the wide-gap semiconductor zinc oxide $(\mathrm{ZnO})$ has been extensively studied in the past decades owing to a large variety of potential applications in light emission, optoelectronic devices, and sensors. ${ }^{1}$ Moreover, the interest for solid-state long-lived quasiparticles as an alternative way for quantum information devices was strongly stimulated by experimental evidences of exciton-polariton condensation and plasmonic lasers. In this context, the direct observation of very long lived optical phonons in $\mathrm{ZnO}$ is really noteworthy. Its vibrational properties have already been investigated, ${ }^{2}$ in particular, under high-pressure $e^{3-5}$ where one can explore the peculiar phonon density of states but $E_{2}$ phonon temperature dependence studies ${ }^{6,7}$ remain partial. We report here Raman spectroscopy measurements on $\mathrm{ZnO}$ crystals grown by the vapor transport method. We first investigated the effects of different thermal treatment conditions; we did obtain a single crystal with an ultra low density of defects. Then, anharmonic effects on the temperature dependence of Raman spectra in the $10-500 \mathrm{~K}$ range are discussed. The linewidth decrease at low temperature is analyzed and interpreted in the light of anharmonic up- and down-conversion processes, unveiling strongly different behaviors for the two $E_{2}$ phonons.

Natural bulk $\mathrm{ZnO}$ adopts wurtzite (WZ) structure with $C 6 \nu$ space group. In fact, up to the second-neighbor shell, the WZ structure is very similar to the zinc-blende (ZB) structure, with two atoms in the unit cell in the ZB and four atoms in the WZ. Therefore, the dispersion curves for the WZ may be deduced from those of the ZB by folding the Brillouin zone along the $\Lambda([111])$ direction. The low- and high-frequency $E_{2}$ zone center modes are the analogous, respectively, to the TA(L) and TO(L) zone edge modes of the ZB structure. This is evidenced by strong similarities in their

\footnotetext{
${ }^{a)}$ Present address: Department of Earth and Planetary Science, University of California-Berkeley, Berkeley, California 94720, USA. Electronic mail: millot.marius@gmail.com.

${ }^{b)}$ Present address: New Materials Department, CIDETEC Centre for Electrochemical Technologies, Parque Tecnológico de San Sebastián Paseo Miramón 196, 20009 Donostia-San Sebastián, Spain
}

physical properties such as a negative Grüneisen parameter. ${ }^{4}$ The two series of $\mathrm{ZnO}$ single crystals used in the present Raman spectroscopy experiments were grown by vapor transport under relatively $\mathrm{Zn}$-rich conditions, using isotopically natural materials. It is worth to mention that series B were grown under richer zinc conditions than series A. More details on the growth technique and strategies applied to modify the $\mathrm{Zn}$ excess in the growth ambient can be found elsewhere. ${ }^{8}$ Unpolarized Raman spectra were performed in backscattering configuration with a confocal Dilor XY 800 triple spectrometer and the $\lambda=514.5 \mathrm{~nm}$ line of an $\mathrm{Ar}^{+}$laser as excitation source. A closed cycle He cryostat was used for the low temperature experiments $(10-300 \mathrm{~K})$, and an electrically heated furnace in the 300-500 K range. As each observed peak is the convolution of the Lorentzian shape of the Raman mode with the Gaussian response function of the spectrometer, we extracted the actual Lorentzian linewidth through Voigt fitting procedure taking into account the spectrometer response function. The Gaussian instrumental width was determined by the measurement of the peak linewidth obtained for a calibrated neon source line. The value used in the following analysis is $0.6 \mathrm{~cm}^{-1}$. Additional spectra have been carried out using a higher resolution T800 Coderg triple spectrometer achieving a $0.5 \mathrm{~cm}^{-1}$ instrumental width.

Our study is focused on Raman mode lineshape analysis; in an ideal harmonic crystal, the lineshape is expected to be infinitesimally narrow, but experimental peaks of real materials exhibit an intrinsic width. On the one hand, the presence of various decay channels shortens the phonon lifetimes by anharmonic processes involving multiple phonon recombinations conserving both energy and crystal momentum. On the other hand, impurities and defects disturb the translation symmetry of the harmonic crystal. Therefore they do modify the Raman linewidth by elastic scattering processes that also contribute to the phonon-lifetime shortening scenario. Finally, the natural isotopic dispersion yields a phonon frequency dispersion that induces an inhomogeneous broadening of the observed Raman modes. Assuming a Boltzmannequation approach, one can take separately into account impurities and anharmonic effects on $\tau$ as $(1 / \tau)=\left(1 / \tau_{\text {imp }}\right)$ 
TABLE I. Annealing conditions and observed linewidth after a $24 \mathrm{~h}$ treatment of the $E_{2}$ (low) $\mathrm{ZnO}$ phonon Raman mode for different samples. The two series of $\mathrm{ZnO}$ single crystals were grown by vapor transport under relatively Zn-rich conditions, using isotopically natural materials: series B were grown under richer zinc conditions than series A.

\begin{tabular}{lcccccccc}
\hline \hline Samples & A1 & A2 & A3 & A4 & B1 & B2 & B3 & B4 \\
\hline Atmosphere & As grown & Vacuum & Oxygen & Zinc & As grown & Vacuum & Oxygen & Zinc \\
Pressure (atm) & & $10^{-5}$ & 2 & 2 & & $10^{-5}$ & 2 & 2 \\
Temperature $\left({ }^{\circ} \mathrm{C}\right)$ & & 900 & 900 & 1000 & & 900 & 900 & 1000 \\
$E_{2}$ (low) FWHM $\left(\mathrm{cm}^{-1}\right)$ & 0.40 & 0.36 & 0.38 & 0.44 & 1.35 & 0.45 & 0.5 & 0.82 \\
\hline \hline
\end{tabular}

$+\left(1 / \tau_{\text {anharmonic }}\right)$. In fact, Heisenberg time-energy uncertainty relation links the measured Raman peak full width at half maximum (FWHM) $\Gamma$ to the phonon lifetime $\tau, \Gamma \propto 1 / \tau$ then we get $\Gamma=\Gamma_{\text {imp }}+\Gamma_{\text {anharmonic }}$. Consequently, to investigate specifically the anharmonic effects, one has to get very high quality samples to avoid as much as possible spurious impurities-related behavior. Hence we have first performed a comparative study of crystalline quality on eight different samples with different thermal treatments measuring the Raman spectra of these different samples at room temperature with special focus on the $E_{2}$ (low) narrow peak (see Table I). The annealing condition efficiency is evidenced by the strong reduction from several $\mathrm{cm}^{-1}$ to a fraction of wavenumber of the $E_{2}$ (low) FWHM. We have then selected the sample A2, for its overwhelming purity as indicated by the $0.36 \mathrm{~cm}^{-1}$ FWHM of the $E_{2}$ (low) phonon at ambient conditions. Therefore, we assumed that elastic scattering on impurities and defects is negligible is this peculiar crystal.

The $\mathrm{ZnO}$ Raman spectra at selected different temperatures are shown on Fig. 1. As it is expected, $E_{2}$ (low), $A_{1}$ (TO), $E_{1}$ (TO), and $E_{2}$ (high) first-order Raman peaks are clearly distinguishable as well as several second-order features corresponding to different wave vectors in the Brillouin zone. The assignment of all peaks is consistent with previous Raman measurements ${ }^{6}$ and phonon dispersion curves obtained by inelastic neutron scattering. ${ }^{9,10}$ The linewidth of the Raman modes was then systematically studied as a function of temperature in the $10-500 \mathrm{~K}$ range; as expected, it is found to increase with temperature (see Fig. 1), e.g., for the $E_{2}$ (high) $\Gamma$ goes from $3.1 \mathrm{~cm}^{-1}$ at $10 \mathrm{~K}$ to $9.95 \mathrm{~cm}^{-1}$ at 473

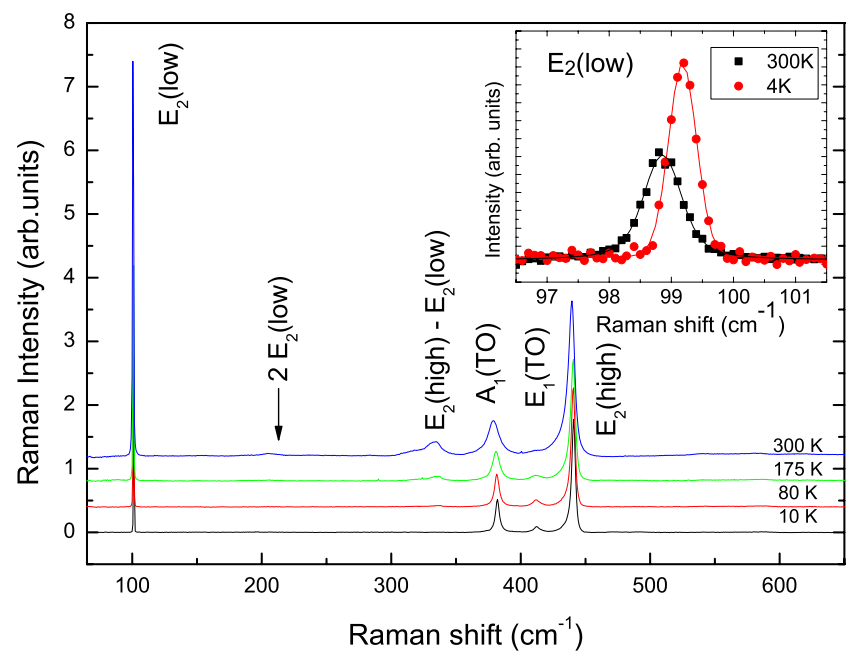

FIG. 1. (Color online) Raman spectra of $\mathrm{ZnO}$ single crystal at selected different temperatures. Inset: zoom on the $E_{2}$ (low) peak measured with the high resolution setup at 4 and $300 \mathrm{~K}$.
$\mathrm{K}$. This broadening is due to the anharmonicity of the lattice vibrations. Let us focus first on the $E_{2}$ (high) mode [see Fig. 2(b)]. This mode, with frequency $\omega_{0}$, is analogous to $\mathrm{Si}$ and Ge optical phonons and thereby, following, ${ }^{11-13}$ we should describe its linewidth temperature dependence taking into account decays into two or three phonons of lower energy: $\Gamma(T)=\Gamma_{0}+A\left(1+\sum_{j=1}^{2} n_{j}\right)+B\left[1+\sum_{j=1}^{3}\left(n_{j}+n_{j}^{2}\right)\right]$ where $n_{j}$ stand for the Boltzmann occupation factor $n_{j}=1 /\left(e^{\hbar \omega_{j} / k_{B} T}-1\right), A$ is the cubic broadening parameter of the phonon line, i.e., the decrease in phonon lifetime $\tau$ due to the decay of the optical phonon into two different phonons and $\mathrm{B}$ is the broadening coefficient owing to the decay into three different phonons. Following ${ }^{11,14}$ we can use the Klemens ansatz: $\omega_{1}=\omega_{2}$ $=\omega_{0} / 2$ for the first term and $\omega_{1}=\omega_{2}=\omega_{2}=\omega_{0} / 3$ for the second term. Another choice is to fix $\omega_{1}=250 \mathrm{~cm}^{-1}$ and $\omega_{2}$ $=190 \mathrm{~cm}^{-1}$ as suggested by ab initio phonon density-ofstates calculations. ${ }^{3,4}$ This model offers a good description of
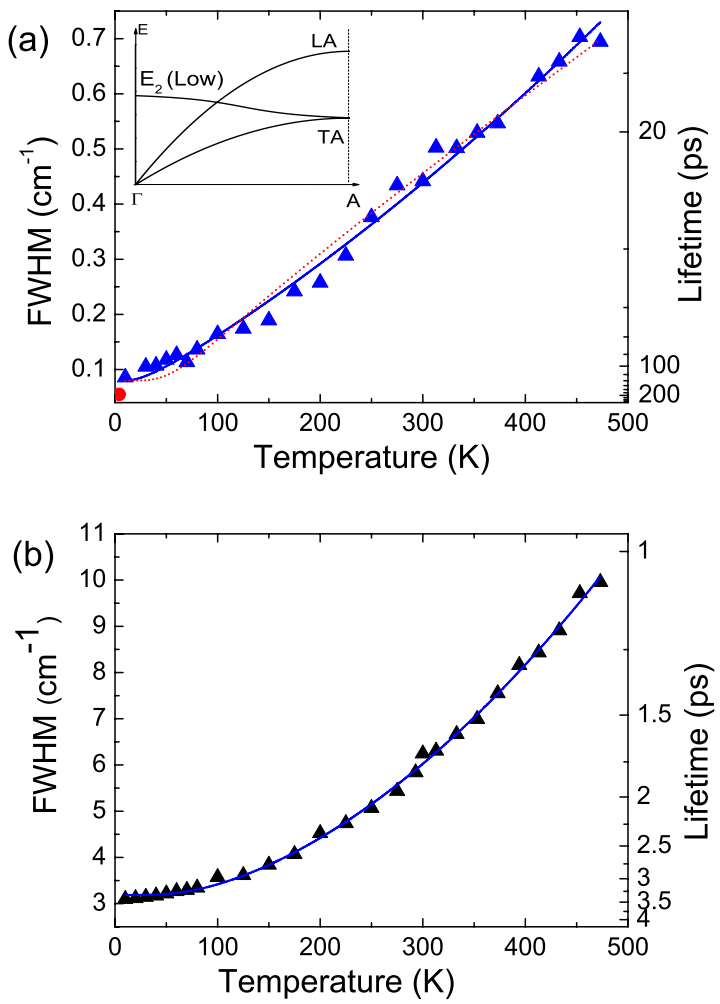

FIG. 2. (Color online) (a) $E_{2}$ (low) linewidth (left scale) and lifetime (right scale) temperature dependence. Both three + four phonon down-conversion (solid line) and three phonon up-conversion (dots) scenarios are in good agreement with experimental data (triangles). Red dot is the high resolution value. Inset: Schematic view of the phonon dispersion curve close to the $\Gamma$ point showing the scarce available decay channels for the $E_{2}$ (low) phonon. (b) Same as (a) but for $E_{2}$ (high). The three+four phonon down-conversion scenario (solid line) accounts well for the observed behavior. 
the experimental behavior as it appears on Fig. 2(b); in fact both fits are superimposed. We find $A=0.55 \mathrm{~cm}^{-1}, B$ $=0.39 \mathrm{~cm}^{-1}$, and $\Gamma_{0}=2.25 \mathrm{~cm}^{-1}$ with the former $\omega_{j}$ set and $A=0.52 \mathrm{~cm}^{-1}, B=0.39 \mathrm{~cm}^{-1}$, and $\Gamma_{0}=2.29 \mathrm{~cm}^{-1}$ with the latter set. However, pure cubic terms, even with upconversion processes proposed in ${ }^{6}$ are not able to fit properly the data. Hence, we unveil that both three and four-phonon anharmonic decay channels are relevant and necessary to describe the temperature dependence of the $E_{2}$ (high) linewidth.

We turn now to the low frequency mode $E_{2}$ (low) [see Fig. 2(a)]. The high coherence, up to $133 \mathrm{ps,} \mathrm{i.e.,} 0.08 \mathrm{~cm}^{-1}$ at $10 \mathrm{~K}$, of this mode reflects the scarce decay channels available owing to the peculiar phonon spectrum [see inset of Fig. 2(a)]. Moreover the acoustical modes density of states is probably particularly low. In fact, this mode is analogous to the Ge acoustic TA mode, characterized by a very long lifetime at low temperature. ${ }^{12}$ Note that at low temperatures the intrinsic linewidth is expected to be inferior than the values shown in Fig. 2(a) due to the finite instrumental resolution. By the way, high resolution additional spectra unravel a lifetime as long as $193 \mathrm{ps}$, i.e., $0.055 \mathrm{~cm}^{-1}$ at $4 \mathrm{~K}$, close to the values $211 \mathrm{ps}$ at $5 \mathrm{~K}$, obtained through impulsive stimulated Raman scattering indirect measurements at $5 \mathrm{~K}^{7}$ Using the previous equation and the Klemens ansatz to fit the observed temperature dependence behavior we get $A=0.040 \mathrm{~cm}^{-1}, B$ $=6.1 \times 10^{-4} \mathrm{~cm}^{-1}$, and $\Gamma_{0}=0.040 \mathrm{~cm}^{-1}$. Hence, the three phonon interaction appears as the dominant lifetime shortening mechanism. In fact, Aku-Leh et al. ${ }^{7}$ suggest from $a b$ initio phonon density-of-states calculations that upconversion are important; one has to consider not only downconversion as $A\left(1+n_{1}+n_{2}\right)$ but also up-conversion terms; $A^{\prime}\left(n_{3}-n_{4}\right)$ to describe the anharmonic properties of this unique phonon. We have then fit the data as $\Gamma(T)=\Gamma_{0}$ $+A^{\prime}\left(n_{3}-n_{4}\right)$ with $\omega_{3}=248 \mathrm{~cm}^{-1}$ and $\omega_{4}=148 \mathrm{~cm}^{-1}$ yielding $A^{\prime}=0.72 \mathrm{~cm}^{-1}$ and $\Gamma_{0}=0.081 \mathrm{~cm}^{-1}$. Hence we unravel that three phonon processes are the dominant anharmonic contribution but both up- and down-conversion could be invoked. In fact, the two extracted $\Gamma_{0}$ values are significantly different because the up-conversion term vanishes at $T=0$. As the $E_{2}$ (low) mode is mainly related to $\mathrm{Zn}$ atom motions, one expects isotopic disorder to contribute to $\Gamma_{0}$; in fact Serrano et al. ${ }^{15}$ estimated $\Gamma_{\text {Isot. }} \sim 0.05 \mathrm{~cm}^{-1}$. We claim that this gives a hint that up-conversion plays here the major role as in this scenario the large $\Gamma_{0}$ would reflect the unavoidable natural isotopic broadening. Finally, the unique anharmonic properties of this mode yield a very long lifetime ultimately limited by the isotopic disorder at low temperature.

To summarize, we have performed a detailed temperature dependent Raman spectroscopy study on zinc oxide crystals. Focusing on $E_{2}$ (high) and $E_{2}$ (low) linewidth we unveil that both three and four-phonon anharmonic decay channels are relevant and necessary to describe the $E_{2}$ (high) behavior whereas three phonon up-conversion processes dominate the $E_{2}$ (low) lifetime shortening properties. An additional high resolution measurement unravels a lifetime as long as $193 \mathrm{ps}$.

Authors thank C. Power, I. Molina, and B. Salas (CESULA) as well as V. Paillard (CEMES-CNRS) for their experimental contribution. Part of this work has been supported by the Spanish government under contract MAT 2007-66129, EuroMagNet under the EU contract $\mathrm{n}^{\circ} 228043$, the FrancoVenezuelian Cooperation Program PCP "Nanotubos de Carbono" and MALTA-Consolider Ingenio 2010 Program.

${ }^{1}$ U. Özgür, Y. I. Alivov, C. Liu, A. Teke, M. A. Reshchikov, S. Dogan, V. Avrutin, S.-J. Cho, and H. Morkoc, J. Appl. Phys. 98, 041301 (2005).

${ }^{2}$ J. M. Calleja and M. Cardona, Phys. Rev. B 16, 3753 (1977).

${ }^{3}$ J. Serrano, F. J. Manjon, A. H. Romero, F. Widulle, R. Lauck, and M. Cardona, Phys. Rev. Lett. 90, 055510 (2003).

${ }^{4}$ J. Serrano, A. H. Romero, F. J. Manjon, R. Lauck, M. Cardona, and A. Rubio, Phys. Rev. B 69, 094306 (2004).

${ }^{5}$ F. Decremps, J. Pellicer-Porres, A. M. Saitta, J.-C. Chervin, and A. Polian, Phys. Rev. B 65, 092101 (2002).

${ }^{6}$ R. Cuscó, E. Alarcon-Llado, J. Ibanez, L. Artus, J. Jimenez, B. Wang, and M. J. Callahan, Phys. Rev. B 75, 165202 (2007).

${ }^{7}$ C. Aku-Leh, J. Zhao, R. Merlin, J. Menendez, and M. Cardona, Phys. Rev. B 71, 205211 (2005).

${ }^{8}$ R. Tena-Zaera, M. Martinez-Tomas, S. Hassani, R. Triboulet, and V. Munoz-Sanjose, J. Cryst. Growth 270, 711 (2004).

${ }^{9}$ A. Hewat, Solid State Commun. 8, 187 (1970).

${ }^{10}$ K. Thoma, B. Dorner, G. Duesing, and W. Wegener, Solid State Commun. 15, 1111 (1974)

${ }^{11}$ J. Menéndez and M. Cardona, Phys. Rev. B 29, 2051 (1984).

${ }^{12}$ J. Kulda, A. Debernardi, M. Cardona, F. de Geuser, and E. E. Haller, Phys. Rev. B 69, 045209 (2004).

${ }^{13}$ H. D. Fuchs, C. H. Grein, R. I. Devlen, J. Kuhl, and M. Cardona, Phys. Rev. B 44, 8633 (1991).

${ }^{14}$ P. G. Klemens, Phys. Rev. 148, 845 (1966).

${ }^{15}$ J. Serrano, F. Widulle, A. H. Romero, A. Rubio, R. Lauck, and M. Cardona, Phys. Status Solidi B 235, 260 (2003). 